\title{
LA SENSIBILIDAD DEL CUIDADOR Y SU IMPORTANCIA PARA PROMOVER UN CUIDADO DE CALIDAD EN LA PRIMERA INFANCIA ${ }^{1}$
}

\section{THE SENSITIVITY OF THE CAREGIVER AND THEIR IMPORTANCE IN PROMOTING QUALITY CARE IN EARLY CHILDHOOD}

\author{
Olga Alicia Carbonell \\ Pontificia Universidad Javeriana, Colombia
}

\begin{abstract}
Palabras clave: apego, cuidador, primera infancia
\end{abstract}
Keywords: attachment, caregiver, early childhood

Para tener una perspectiva más amplia de lo que se entiende hoy por calidad del cuidado infantil, es relevante asumir una mirada histórica sobre algunas representaciones de infancia y sus prácticas de cuidado. El historiador francés Ariès (1987), en su libro "El Niño y la Vida Familiar en el Antiguo Régimen", muestra que el estatus de infancia de la época no corresponde a como en la actualidad se piensa lo que es un niño o una niña desde una perspectiva de sujeto de derechos, o desde la infancia como una etapa del desarrollo humano. En siglos anteriores, los niños y los jóvenes compartían los modos de vestir, las actividades, el lenguaje y la vida cotidiana del mundo adulto. El sentimiento de la infancia era demasiado frágil debido a que muchos niños morían muy temprano.

Así mismo, si se considera el interesante trabajo sobre la historia de la infancia de De Mause (1974) "La Evolución de la Infancia", se encuentra como él mismo dice, no la infancia novelada, deformada de historiadores y literatos, por biógrafos oficiales, tales como Héroard médico en la infancia de Luis XIII, sino una infancia desde una historia que "debe desentrañar la realidad de las condiciones sociales de otras épocas" (p. 3), y no legitimar las prácticas sociales a través de datos ocultos, suavizados y/o deformados. Este autor señala que el maltrato, el abuso, el infanticidio, el trabajo infantil y el abandono de los niños no son fenómenos sociales recientes, sino que eran prácticas de crianza comunes y aceptadas desde la Antigüedad. El castigo corporal extremo como otras formas disciplinarias utilizadas con los niños (fajarlos, aterrorizarlos con máscaras, historias y personajes), así como el control social sobre el cuerpo del niño, tanto en la evacuación y el sexo, han estado socialmente legitimados a lo largo de la historia de la humanidad.

En este sentido Casas (1998), retoma el concepto de representación social como el producto del consenso colectivo de un grupo humano en un momento histórico determinado respecto a un fenómeno social. En este caso refiriéndose a la representación de infancia, afirma que no es una realidad objetiva y universal, puesto que cada sociedad define explícita o implícitamente lo que es infancia. Estas representaciones son construcciones sociales y por tanto, son susceptibles de sufrir cambios y modificaciones en una misma cultura y en distintos momentos históricos. Sin embargo, señala que debido a que son creencias compartidas y legitimadas por la mayoría del grupo social y que se transmiten intergeneracionalmente, no se cuestionan y se invisibilizan tanto en la representación como en las prácticas sociales.

Las concepciones de infancia que hemos presentado, distan mucho de las modernas concepciones de infancia de los niños y las niñas sujetos de derechos y como agentes sociales y políticos, en contraposición a las posturas de los niños beneficiarios de caridad y receptores pasivos (Fondo de las Naciones Unidas para la Infancia [UNICEF], 2009), que hoy a través de la Convención de los Derechos del Niño (Naciones Unidas,1989) y de múltiples instituciones gubernamentales y no gubernamentales, se ha venido propagando en los 193 países firmantes de la convención. No obstante, los informes mundiales sobre la infancia de UNICEF de los últimos 
años, muestran que los derechos de millones de niños y niñas del mundo y de América Latina son vulnerados de manera sistemática.

Hoy se señala que el cumplimiento de los derechos de los niños y las niñas es una corresponsabilidad del Estado, la familia y la comunidad (Instituto Colombiano de Bienestar Familiar [ICBF], 2006), sin embargo, la transformación de actitudes en relación con la infancia, no es un asunto fácil, porque gran parte de la población posee concepciones de infancia que son contrarias al niño y a la niña como sujeto de derechos, y cree y legítima en su discurso y en sus prácticas cotidianas actitudes autoritarias, centradas desde la perspectiva adulta, que no le concede al niño y la niña su derecho a la autonomía y a la participación.

Muchas de las prácticas de crianza y cuidado actuales son maltratadoras y negadoras de los derechos de los niños y las niñas y están atravesadas por discursos culturales, algunos que todavía escuchamos en ciertos padres o madres, donde los niños son concebidos como "propiedad de los padres" y por eso pueden hacer con ellos lo que consideren coherente con sus ideas y experiencias propias sobre la crianza; como ser abusivos y llegar al maltrato infantil para disciplinarlos (Asociación Colombiana para la Defensa del Menor Maltratado, 1993). Este último, que ha existido siempre en múltiples manifestaciones, en la historia de la humanidad, como ya se mencionaba, se cuestiona públicamente en Estados Unidos en los inicios de la década de los años sesenta, por parte del pediatra Kempe y sus colaboradores refiriéndose al "Síndrome del Niño Golpeado". Este síndrome se encontraba legitimado en el seno de dicha sociedad como algo cotidiano en el mundo privado de la familia y público de la escuela, por diversas razones sociales, culturales y religiosas, entre otras (como se citó en Gershoff, 2002). No obstante, la sociedad norteamericana y sus medios de comunicación en la década de los años sesenta se encontraban sensibles y receptivos a la defensa de los derechos civiles de la población. Esto dio lugar a empezar a gestar una nueva representación de infancia, que implicó una nueva legislación y presupuestos para entender y prevenir el maltrato infantil (Casas, 1998).

La forma más visible del maltrato infantil, es el maltrato físico, pues implica en la mayoría de los casos huellas físicas (Howe, 2005; Montoya, 1993). Sin embargo, existen otras formas como la negligencia o el abandono físico y psicológico, el abuso sexual, unas más fáciles y otras más difíciles de identificar. También, está el maltrato psicológico que es aun más difícil de evaluar, pues no deja huella visible (Howe, 2005; Isaza, 1993). Otra modalidad del maltrato que fácilmente pasa desapercibido, es el maltrato institucional, donde los niños y las niñas, pueden verse maltratados a través de un trato despersonalizado que en ocasiones llega a la negligencia en la prestación de servicios por parte de los profesionales o de sistemas privados y públicos tales como la salud, donde a los niños no se les dan explicaciones, ni se les escucha sus necesidades, no son interlocutores activos, sino receptivos (Casas, 1998). No obstante, desde una perspectiva ecológica del desarrollo es importante entender el maltrato infantil desde múltiples contextos, unos más próximos como las relaciones adulto-niño, que es el contexto de cuidado e interactivo inmediato, donde es relevante considerar la transmisión intergeneracional de estos patrones de crianza, así como el contexto más amplio donde está la comunidad y la cultura (Howe, 2005; Belsky, 1993).

Existen otras prácticas de crianza infantil que utilizan formas más sutiles y altamente generalizadas como el castigo físico o llamado por algunos autores como castigo corporal para no usar un lenguaje que camufla una dura realidad infantil (Gershoff, 2002). Esta forma de castigo es definido por Strauss como, el uso de la fuerza física con la intención de causar a un niño dolor aunque no daño, con el propósito de corregir o controlar el comportamiento del niño (como se citó en Gershoff, 2002); administrado por los padres o adultos responsables de los niños y niñas y aplicado como forma de corrección razonable, moderada y legal (Gialdino, 2007).

El Comité de los Derechos del Niño, en junio del 2006 se pronuncia contra el castigo corporal y otras formas de castigos degradantes y crueles, en el artículo 19. Posteriormente, la Comisión Interamericana de Derechos Humanos $(\mathrm{CIDH})$ en la Relatoría sobre derechos de la niñez de la OEA en 2009, en su informe sobre el castigo corporal y los derechos humanos de las niñas, niños y adolescentes, reconoce la gravedad y seriedad de esta práctica como violatoria de la dignidad de los niños y niñas, recomendando a los países miembros acciones concretas como adoptar políticas, leyes, medidas preventivas y educativas que prohíban a los padres y adultos responsables, tanto en 
el ámbito privado (hogar) y público (escuelas, correccionales) usar el castigo corporal como un medio para disciplinar a los niños y niñas.

Actualmente sólo 24 países del mundo prohíben legalmente el castigo corporal -incluso en el hogar-, de los cuales sólo tres son miembros de la OEA, Uruguay, Venezuela y Costa Rica. Todavía hoy en muchos países miembros de la OEA, el castigo corporal permanece en el código penal como método disciplinario. Esta práctica de socialización y control, es utilizada de forma generalizada por los adultos responsables de su cuidado y protección, especialmente con los niños en la primera infancia y en los años escolares, sin que se considere abusiva. Es violencia privada, legal y socialmente aceptada y tolerada (Gershoff, 2002; Gialdino, 2007).

Como ha sido posible observar la infancia está históricamente cargada con formas permanentes de violencia dirigida hacia los niños y las niñas. Por tanto, estamos frente a un gran reto, que no solamente debe ser controlado a través de las legislaciones de los países, sino a través de la supervisión, vigilancia y diversas formas de transformación cultural de los discursos y prácticas de cuidado a la infancia por parte del Estado, las familias y la sociedad civil (Gialdino, 2007). Es necesario que la nueva representación de infancia de la convención de los derechos del niño, el niño como sujeto de derechos, pase de ser un ideal ético-político de la humanidad a una realidad cotidiana en la vida de los niños y las niñas.

Desde la psicología, y específicamente desde la teoría del apego (Bowlby, 1969/1993), a partir de estudios realizados en distintos contextos culturales (Ainsworth, Blehar, Waters, \& Wall,1978), se ha encontrado el papel tan importante que juega la calidad del cuidado por parte de la madre y de aquellos adultos encargados de cuidar a los bebés y niños pequeños, en el desarrollo infantil.

La calidad del cuidado, se refiere a los comportamientos y estrategias que usan los cuidadores principales y en particular la madre, para cuidar, proteger y garantizar la supervivencia de los bebés y los niños pequeños. Se consideran cuidadores principales, todos aquellos adultos familiares y no familiares, padre, abuelos, tíos, niñeras, etc., que apoyan el cuidado, aunque no en forma exclusiva, así como maestras preescolares o de jardines infantiles, vecinos y demás adultos.
Las observaciones pioneras de díadas madre-bebé en sus interacciones en ambientes naturales de la vida cotidiana, principalmente en el hogar, en el primer año de vida de Mary Ainsworth, en Uganda, África (Ainsworth, 1967) y posteriormente en Baltimore, Estados Unidos en las décadas de los años 60 y 70 (Ainsworth et al.,1978), le permitieron elaborar una conceptualización del cuidado temprano, descrita a partir de un continuo de cuatro características del comportamiento materno, que va desde lo más positivo hasta lo más negativo: 1) Aceptación-rechazo, 2) cooperación-interferencia, 3) accesibilidad-ignorar y 4) sensibilidad-insensibilidad (Ainsworth, Bell \& Stayton, 1974).

La primera característica comportamental, aceptación-rechazo, hace referencia que para toda madre o cuidador principal existen sentimientos positivos y negativos frente al cuidado de un bebé o niño pequeño, debido a las exigencias y demandas que esto implica. En el polo o extremo positivo se encuentran los sentimientos de amor, aceptación, ternura, protección, goce compartido y cualquier otro tipo de reacción positiva generada por el bebé o niño pequeño. En el otro extremo, están los sentimientos de rabia, resentimiento, irritación y rechazo, entre otras emociones negativas generadas por el comportamiento del niño o las demandas de cuidado exigidas por la crianza. Se parte del supuesto que en toda relación madre o cuidador-bebé o niño pequeño, hay sentimientos positivos y negativos, es decir, algún grado de ambivalencia, no obstante, la cuestión está en qué medida la madre o el cuidador principal es capaz de equilibrarlos e integrarlos, de forma que los aspectos negativos no permeen y primen en la relación con el niño o la niña.

En cuanto a la segunda característica comportamental cooperación-interferencia, ésta hace referencia en el extremo positivo a la capacidad del cuidador en sincronizar o sintonizarse afectivamente y en el comportamiento con el niño, lo cual implica considerarlo como un ser autónomo, activo, cuyos deseos, sentimientos y actividades son validados y respetados por el adulto cuidador. En el otro extremo, la interferencia, está el adulto cuidador que no respeta las iniciativas o autonomía del niño, que niega las necesidades, los deseos, sentimientos y actividades de éste, pues impone su voluntad y deseos de adulto sobre los del niño. En muchos casos, éstos adultos cuidadores consideran que 
el niño o la niña es de su propiedad, o creen que los niños deben someterse a la voluntad del adulto, por estos motivos, entre otros, los controlan, los entrenan y los castigan para que se comporten de acuerdo a sus expectativas de lo que considera que es el comportamiento adecuado, sin escuchar la voz del niño.

De acuerdo a la tercera característica del comportamiento del cuidador, accesibilidadignorar, ésta se refiere en el extremo positivo a la disponibilidad física y psicológica del adulto cuidador respecto a las necesidades del bebé o del niño pequeño. Es decir, permitir la cercanía y contacto físico, está dispuesto emocionalmente para el niño, mostrar gusto por estar y compartir con él. En el extremo negativo, el adulto cuidador que ignora las necesidades y comunicaciones del niño, por estar focalizado en sus propias necesidades y preocupaciones, no está en la mayoría de las ocasiones disponible emocionalmente para éste. Esto puede darse, en los casos de madres deprimidas (Shonkoff \& Phillips, 2000; Spieker, Nelson, DeKlyen, \& Staerkel, 2007; Tronick, \& Weinberg, 1997; Zeanah, Boris, \& Larrieu, 1997). En las formas más extremas, nos encontramos los comportamientos negligentes físicos y psicológicos por parte del adulto cuidador, que son considerados hoy, formas de maltrato infantil (Howe, 2005).

La cuarta característica del comportamiento del cuidador es la sensibilidad-insensibilidad del cuidador a las comunicaciones del bebé o del niño pequeño. Esta se refiere en el componente positivo a la habilidad de la madre o cuidador principal, a estar alerta a las señales comunicativas del niño, interpretarlas adecuadamente y responderlas pronta y correctamente. Por el contrario, en el extremo opuesto del continuo, se encuentra la madre o cuidador, que ignora las comunicaciones del bebé o niño pequeño, las interpreta incorrectamente, suponiendo muchas veces atribuciones e intencionalidades negativas por parte del bebé o del niño (deseos de molestar o manipular al adulto). Por tanto, no responde prontamente a las comunicaciones y necesidades del niño, basada en argumentos culturales tales como "darle al niño todo lo que quiere es malcriarlo", permitiendo que señales negativas como el llanto se intensifiquen. Es importante aclarar que una madre o un cuidador insensible, no es aquel que necesariamente su respuesta al niño o la niña es hostil o desagradable. La insensibilidad del cuidado ocurre cuando el cuidador falla o no logra leerle e interpretar adecuadamente los estados emocionales o metas del bebé o niño pequeño, por lo tanto, no logra ayudarlo para que obtenga de nuevo un estado emocional positivo, por ejemplo si está ansioso y llorando, no logra calmarlo. El cuidado poco sensible, le enseña al niño que sus comunicaciones no son efectivas o aun peor en algunas ocasiones son contraproducentes, por ejemplo cuando solicita algo y la respuesta del cuidador es rechazarlo. Así mismo, la respuesta inconsistente del cuidador poco sensible de manera recurrente, a sus demandas y señales comunicativas, lo lleva a sentir que el mundo es impredecible, no tiene control sobre éste y por tanto, no se le favorece un sentimiento de eficacia en el actuar sobre el mundo (Colin, 1996).

Tampoco es posible igualar sensibilidad en el cuidado con calidez o amor, son conceptos distintos (Seifer y Schiller, 1995), ya que el comportamiento sensible de cuidado, es producto de una relación reciproca y coordinada donde ambos interlocutores, adulto cuidador y niño, están en una relación de cooperación y sintonizada, tanto emocional como comportamentalmente. No obstante, las relaciones vinculares madre o cuidador sensible con el niño o la niña están inmersas en un clima emocional $\mathrm{y}$ afectivo positivo.

En dos estudios recientes, uno de ellos realizado en Colombia y el otro en Estados Unidos, con diadas de niños preescolares y sus mamás, se han encontrado también asociaciones significativas entre tres comportamientos maternos de cuidado con la seguridad emocional de los niños: 1) interacciones armoniosas entre la madre y el niño, 2) apoyo materno al uso de la base segura para explorar el mundo físico y social por parte del niño o niña y 3) supervisar y monitorear al niño (Plata, Pérez, Peña, Carbonell \& Posada, 2011; Posada, Kaloustian, Richmond, \& Moreno, 2007).

Estudios durante las últimas cuatro décadas por parte de múltiples investigadores de la teoría del apego en distintos países del mundo y en diferentes contextos sociales y situaciones, han encontrado una asociación significativa entre la sensibilidad del cuidador y la seguridad emocional del niño o niña (van ljzendoorn \& Sagi, 2008). Estos estudios han permitido determinar el papel clave que juega el cuidador principal como una base de seguridad desde la cual el niño o la niña pequeña organiza su comportamiento, logra seguridad, explora y aprende sobre el ambiente, tanto físico como 
social (Ainsworth, 1967; Bowlby, 1969/1993; Posada, Carbonell, Alzate, \& Plata, 2004). Especialmente los niños en las situaciones de estrés, tales como hambre, sueño, cansancio, temor, enfermedad, usan al cuidador principal como refugio de seguridad (Sroufe \& Waters, 1977).

Las investigaciones transculturales (van ljzendoorn \& Sagi-Schwartz, 2008) han mostrado los siguientes hallazgos principales:

1) En los países occidentales todos los bebés cuando se les da la oportunidad se apegan afectivamente a uno o más cuidadores del ambiente familiar (padre, madre, abuela, etc.) o cuidadores no familiares, excepto, si poseen un daño neurofisiológico (retardo mental extremo). 2) En las sociedades occidentales la mayoría de los bebés y los niños pequeños muestran apego seguro. Es decir, usan la(s) figura(s) de apego como base de seguridad para explorar el ambiente y como refugio de seguridad en situaciones de estrés. Un porcentaje cercano al $40 \%$ de los bebés y niños pequeños manifiestan apegos inseguros. Los apegos seguros varían considerablemente dentro de las poblaciones y grupos dentro de la misma cultura. Los niños seguros en situaciones de estrés son más fáciles de tranquilizar fisiológicamente y recurren a los adultos cuando los necesitan. 3) La seguridad del apego depende de la calidad de cuidado infantil, especialmente, si los cuidadores dan respuestas sensibles y prontas a las señales y comunicaciones del bebé y de los niños pequeños. 4) Diversos estudios han mostrado que los niños en la primera infancia, en los años preescolares y en la escuela primaria con relaciones de apego seguras comparados con aquellos con relaciones de apego inseguras, presentan mayores competencias en diversas áreas del desarrollo, tales como: mayor capacidad para regular sus emociones negativas (rabia, temor y formas constructivas de enfrentar el estrés), establecer relaciones sociales satisfactorias con compañeros y profesoras, mejor autoconcepto y mayores desarrollos en habilidades cognitivas.

Por otra parte, los niños y las niñas que viven en situación de pobreza, se enfrentan a muchos estresores en sus vidas por factores adversos psicosociales y económicos. Algunos estudios han mostrado que los padres y los cuidadores actúan como mediadores de los efectos de la pobreza, en un sentido positivo o negativo para el desarrollo infantil (Shonkoff \& Phillips, 2000). Investigaciones en ambientes familiares con estresores económicos y psicológicos, han encontrado en niños preescolares, que aquellas madres que les proveían un ambiente del hogar estructurado y sensible a las necesidades de los niños, éstos se mostraban más competentes en habilidades cognitivas y lingüísticas comparados con aquellos que no contaban con dicho ambiente de cuidado (Pianta et al., como se citó en Egeland, Carlson, \& Sroufe, 1993). Así mismo, otras investigaciones han mostrado que los dos factores protectores más importantes hallados con niños competentes en el preescolar en situaciones de adversidad social y económica, son: poseer un apego seguro con la madre entre los 12 a 18 meses de edad $y$ tener un cuidado sensible y emocionalmente responsivo a las necesidades del niño, por parte de ésta. De esta manera, los comportamientos de las madres los protegen de los efectos del estrés, dándoles un cuidado de buena calidad (Egeland et al., 1993).

Los hallazgos de algunos estudios sugieren que las condiciones de riesgo severo están mediadas en parte por el cuidado temprano que el niño o la niña recibe de sus padres o de otros cuidadores. En el sentido contrario, los niños que han sufrido de manera crónica un cuidado emocionalmente insensible, muestran dificultades severas en todas sus áreas del desarrollo, no solo en el momento presente, sino en etapas posteriores de su vida (Dozier, Lindhiem, \& Ackerman, 2007; Egeland et al., 1993; Shonkoff \& Phillips, 2000; Zeanah \& Smyke, 2007).

Así mismo, en el estudio longitudinal de Minnesota (Sroufe, Egeland, Carlson, \& Collins, 2005) hallaron que el tener una relación emocional responsiva en la niñez por parte de padres adoptantes o de un pariente cercano, parece ser un factor que explica en parte porque algunas madres que fueron maltratadas $(30 \%)$, posteriormente cuando fueron madres estuvieron en capacidad de romper el ciclo intergeneracional del maltrato, dándoles a sus propios hijos un cuidado sensible. Sin embargo, los resultados de este estudio longitudinal señalan que de las madres maltratadas en su infancia, que no tuvieron cuidadores alternativos sensibles, el $40 \%$ de ellas, maltrataban a sus propios hijos, y $30 \%$ les proveían un cuidado limítrofe con el abuso.

Desde esta perspectiva, se entiende la resiliencia como un proceso, en el cual una persona desarrolla la capacidad de lograr resultados positivos en su desarrollo, a pesar de tener circunstancias adversas o amenazantes a través del tiempo, si crece y se desarrolla en un 
ambiente de cuidado que le dé apoyo emocional y considere sus necesidades (Delage, 2010; Egeland et al., 1993).

De esta manera, cualquier cuidador del medio familiar o social, juega un papel protector en el desarrollo de la persona. En este sentido, la resiliencia es resultado de las transacciones y experiencias internas y externas del niño con un ambiente, más que el resultado de características propias de la persona (Delage, 2010; Egeland et al., 1993).

En este mismo orden de ideas, la teoría del apego es una conceptualización coherente con una concepción del niño y la niña como sujetos de derechos, pues hablar de relaciones de cuidado de calidad, implica pensar los ambientes de cuidado, tanto en sus aspectos físicos y psicológicos desde el concepto de sensibilidad del cuidador. Es decir, un cuidador capaz de ver las cosas desde el punto de vista del niño o de la niña, y que negocia de manera flexible las necesidades y metas en conflicto. También, el cuidado sensible implica ajustarse a los estados emocionales y al momento evolutivo del niño y de la niña, favoreciendo ambientes de cuidado amorosos y cálidos (Carbonell, Posada, Plata y Méndez, 2005).

La comprensión y aplicabilidad en la política pública y en los contextos familiares y comunitarios, del concepto de sensibilidad en la crianza, a partir de la corresponsabilidad Estado, familia y comunidad, tiene implicaciones muy importantes para el cumplimiento de la Convención de los Derechos del Niño, pues, asume al niño y a la niña como sujetos de derechos como interlocutores activos, con necesidades e intereses propios. En consecuencia, modifica la concepción del niño o la niña como seres pasivos y dependientes de la voluntad del adulto cuidador. Esta nueva perspectiva frente al niño y la niña, es un precursor para que desde el inicio de la vida, se les reconozca los derechos a la supervivencia y desarrollo, la protección y promoción, y particularmente a la participación y la autonomía (Carbonell et al., 2005).

Por tanto, es un compromiso y gran reto de los que trabajan a favor de la infancia como gestores conscientes, el diseño de intervenciones que motiven la transformación cultural de aquellas representaciones y prácticas de cuidado infantil no favorecedoras del desarrollo de los niños y las niñas como sujetos de derechos, tanto en el medio familiar y escolar, que son los primeros núcleos de convivencia social y de experiencia emocional de los niños y las niñas. Por tanto, es corresponsabilidad de la sociedad civil ser garantes de los derechos de los niños y las niñas desde los múltiples sectores (nacional, regional, local y familiar). Así mismo, existe una responsabilidad ética y social desde la psicología y demás áreas del conocimiento de abordar las problemáticas de la infancia y buscar soluciones para lograr una calidad de vida acorde con los presupuestos de la Convención de los Derechos del Niño.

\section{Referencias}

Ainsworth, M. D. S. (1967). Infancy in Uganda. Baltimore: Johns Hopkins University Press.

Ainsworth, M. D. S., Bell, S. M., \& Staton, D. J. (1974). Infant-mother attachment and social development: "Socialization" as a product of reciprocal responsiveness to signals. En M. P.M. Richards (Ed.), The integration of a child in the social world (pp.99-135). London: Cambridge University Press.

Ainsworth, M. D. S., Blehar, M. C., Waters, E., \& Wall, S. (1978). Patterns of attachment: A psychological study of the strange situation. Hillsdale, $\mathrm{N} \mathrm{J}$ : Erlbaum.

Ariès, Ph. (1987). El niño y la vida familiar en el antiguo régimen. Madrid: Taurus.

Asociación Colombiana para la Defensa del Menor Maltratado (1993). El drama de la niñez maltratada en Colombia. Bogotá: Guadalupe.

Belsky, J. (1993). Etiology of child maltreatment: A developmental ecological analysis.Psychological Bulletin, 114(3), 413-434. doi: 10.1037/0033-2909.114.3.413.

Bowlby, J. (1993). El Vínculo Afectivo. Barcelona: Editorial Paídos. (Trabajo original publicado en 1969).

Carbonell, O.A., Posada, G., Plata, S.J., y Mendez, S. (2005). Las relaciones vinculares: Un contexto para potenciar el derecho al bienestar de niños y niñas. Cuadernos de Psicologia, 1, 31-38.

Casas, F. (1998). Infancia: Perspectivas psicosociales. Barcelona: Paídos.

Colin, V. L. (1996). Human Attachment. New York: McGraw-Hill. Instituto Colombiano de Bienestar Familiar (2006). Colombia por la primera infancia. Política pública por los niños y niñas, desde la gestación hasta los 6 años. Recuperado de http://www.mineducacion.gov.co/primerainfancia/1739/article-177828.html

Comisión Interamericana de Derechos Humanos (2009). Informe sobre el castigo corporal y los derechos humanos de las niñas, niños y adolescentes. Recuperado de http://www.cidh.oas.org/Ninez/CastigoCorporal2009/ CastigoCorporal.1.htm

Delage, M. (2010). La resiliencia familiar. El nicho familiar y la superación de las heridas. Barcelona: Gedisa.

De Mause, LI. (1974). La evolución de la infancia. Recuperado de http://www.psicodinamicajlc.com/articulos/ evolucion_infancia.html

Dozier, M., Lind̄hiem, \& Ackerman, J. P. (2007). Attachment and biobehavioral cath-up. An intervention targeting empirically identified needs of foster infants. En Lisa J. Berlin, Yair Ziv, Lisa Amaya-Jackson, \& Mark T. Greenberg (Eds.), Enhancing Early Attachments: Theory, research, intervention, and policy. (pp. 178-194). New York: The Guilford Press.

Egeland, B., Carlson, E., \& Sroufe, A. (1993). Resilience as process. Development and Psychopathology, 5, 517528. doi:10.1017/S0954579400006131. 
Gershoff, E. T. (2002). Corporal punishment by parents and associated child behaviors and experiences: A metaanalytic and theoretical review. Psychological Bulletin, 128, 539-579. doi:10.1037//0033-2909.128.4.539.

Gialdino, R.E. (2007). El derecho de los niños a la protección de los castigos corporales y otras formas de castigos crueles y degradantes. Recuperado de http://biblio. juridicas.unam.mx/revistas/resulart.htm

Howe, D. (2005). Child abuse and neglect. Attachment, development and intervention. New York: Palgrave Macmillan.

Isaza, C. C. (1993). Aspectos psicológicos del maltrato infantil. En Asociación Colombiana para la defensa del menor maltratado (Eds.), El drama de la niñez maltratada en Colombia (pp. 23-24).Bogotá: Guadalupe

Montoya, C.A. (1993). Aproximación al diagnóstico del maltrato. En Asociación Colombiana para la Defensa del Menor Maltratado (Eds.), El drama de la niñez maltratada en Colombia. Bogotá:Guadalupe.

Naciones Unidas (1989). Convención sobre los Derechos del Niño. Recuperado de http://www2.ohchr.org/spanish/law/crc.htm

Plata, S. J, Pérez, J., Peña, P. A Carbonelll, O. A, \& Posada, G. (2011). Maternal Sensitivity and Preschoolers' Secure Base Behavior. Poster presentado en el congreso bi-annual Society for Research in Child Development. Montreal, Canada.

Posada, G., Carbonell, O.A, Alzate, G., \& Plata, S.J. (2004). Through Colombian lenses: Ethnographic and conventional analyses of maternal care and their associations with secure base behavior. Developmental Psychology, 40, 508-518. doi: 10.1037/0012-1649.40.4.508

Posada, G., Kaloustian, G., Richmond, M. K., \& Moreno, A. J. (2007). Maternal secure base support and preschoolers' secure base behavior in natural environments. Attachment \& Human Development, 9, 1-19. doi: 10.1080/14616730701712316.

Seifer, R., \& Schiller, M. (1995). The role of parenting sensitivity, infant temperament, and dyadic interaction in attachment theory and assessment. Monographs of the Society for Research in Child Development, 60 , 2-3,146-174. doi:10.2307/1166176.

Spieker, S., Nelson, D., DeKlyen, M., \& Staerkel, F. (2007). Enhancing early attachment in the context of Early Head Star. Can programs emphasizing infant-mother attachment in low-income families? En L. J. Berlin, Y. Ziv, L. Amaya-Jackson, \& M. T. Greenberg (Eds.), Enhancing Early Attachments: Theory, research, intervention, and policy. (pp. 195-216). New York: The Guilford Press.
Shonkoff, J.P. \& Phillips, D.A. (2000). From neurons to neighborhoods. The Science or Early Childhood Development. Washington, D.C.: National Academic Press.

Sroufe, L. A., Egeland, B., Carlson, E. and Collins, W. A. (2005). The development of the person: The Minnesota study of risk and adaptation from birth to adulthood, New York: Guilford.

Sroufe, A., \& Waters, E. (1977). Attachment as an organizational construct. Child Development, 48, 1184-1199. doi:10.2307/1128475

Fondo de las Naciones Unidas para la Infancia. (2009). Estado mundial de la infancia. Edición Especial. Conmemoración de los 20 años de la Convención sobre los derechos del niño. Recuperado de http://www.unicef. org/spanish/sowc2013/files/SOWC_SpecEd_CRC_ MainReport_SP_100109.pdf

Tronick, E. Z., \& Weinberg, M. K. (1997). Depressed mothers and infants: Failure to form dyadic states of consciousness. En L. Murray \& P. J. Cooper (Eds.), Postpartum depression and child development (pp.54-81). New York: Guilford Press.

van ljzendoorn, M. H. \& Sagi-Schwartz, A. (2008). Crosscultural patterns of attachment: Universal and contextual dimensions. The measurement of attachment security and related constructs in infancy and early childhood. Edn J. Cassidy \& P. R. Shaver. Handbook of Attachment: Theory, research, and clinical applications. pp. (880-905). New York: The Guilford Press.

Zeanah, C.H., Boris, N. W., \& Larrieu, J. A. (1997). Infant development and developmental risk: A review of the past 10 years. Journal of the American Academy of Child and Adolescent Psychiatry, 36, 2, 165-178. doi:10.1097/00004583-199702000-00007.

Zeanah, C. H., \& Smyke, A. T. (2007). Building Attachment Relationships Following Maltreatment and Severe Deprivation. En L. J. Berlin, Y. Ziv, L. Amaya-Jackson, \& M. T. Greenberg (Eds.), Enhancing Early Attachments: Theory, research, intervention, and policy. (pp. 195-216). New York: The Guilford Press.

1 (N. de la Ed.) Conferencia dictada por la Dra. Olga Alicia Carbonell el 13 de mayo de 2013 (revisada por la autora). Profesora invitada por el Departamento de Psicología del Desarrollo y Educación de la Facultad de Psicología de la Universidad Católica del Uruguay.

La Prof. Olga Alicia Carbonell Blanco. Ph.D, de la Facultad de Psicología de la Pontificia Universidad Javeriana de Colombia, es una destacada académica que tiene bajo su responsabilidad diversas cátedras e imparte cursos y conferencias en Universidades de distintos países.

Egresada como Ph.D. de la Universidad de Purdue, USA; tiene estudios de postrado en Psicología Clínica y Psicopatología, asì como de especialización en Desarrollo, en la Universidad de Paris V, Francia. Además de ser Miembro fundadora de la Red Iberoamericana de Apego (RIA), es Miembro de la Society for Research in Child Development (SCRD). Ha recibido diversos premios y distinciones académicas, entre ellos: el Bowlby-Ainsworth award en el año 2011; el Jacob travel-lab visit award de la Society for Research in Child Development, en el 2010. Investigadora Principal responsable de proyectos en el campo de las relaciones familiares y la infancia. Cuenta con publicaciones en revistas científicas y libros, que son de referencia en su área de experta.

Para citar este artículo:

Carbonell, O. A. (2013). La sensibilidad del cuidador y su importancia para promover un cuidado de calidad en la primera infancia. Ciencias Psicológicas VII (2): 201 - 207. 